\title{
A multicenter prospective randomized trial of a second-generation anastomotic device in coronary artery bypass surgery
}

\author{
Lars Wiklund, MD, ${ }^{\mathrm{a}}$ Marek Setina, MD, ${ }^{\mathrm{b}}$ Katherine Tsang, $\mathrm{MS},{ }^{\mathrm{c}}$ Robert Cusimano, MD, MSc, ${ }^{\mathrm{c}}$ \\ and Terrence $\mathrm{Yau}, \mathrm{MD}, \mathrm{MSc}^{\mathrm{c}}$
}

Objective: Our objective was to perform a prospective randomized trial to evaluate the clinical and angiographic outcomes of a second-generation anastomotic device used for saphenous vein grafts.

\begin{abstract}
Methods: Patients undergoing nonemergency isolated coronary artery bypass grafting at 3 centers from August 2003 to December 2004 with at least 2 saphenous vein grafts were included. The proximal anastomoses were randomized, within each patient, to be constructed by the connector or by suture. One-year graft patency was evaluated by coronary angiography, magnetic resonance imaging, or computed tomography and analyzed on an intent-to-treat basis.
\end{abstract}

\begin{abstract}
Results: A total of 151 patients ( $65 \pm 9$ years, $87 \%$ male) who met inclusion/exclusion criteria were enrolled in the study and were analyzed. A total of 489 grafts were constructed ( $3.2 \pm 0.5$ grafts per patient), including 327 vein grafts randomized to the connector $(n=162)$ or suture $(n=165)$. In 162 connector grafts, 151 devices were successfully implanted. Technical issues required explantation of 11 devices intraoperatively. Patency was evaluated in $120(81 \%)$ patients with 260 study grafts. Seventy-four patients with 161 grafts were evaluated by coronary angiography, 31 patients with 69 grafts by magnetic resonance imaging, and 15 patients with 30 grafts by computed tomography. The 1-year patency rate for study grafts constructed with the anastomotic connector was $92.2 \%$ (118/128) and for hand-sutured grafts, 91.7\% (121/132).

Conclusions: This prospective multicenter randomized controlled trial demonstrated good in-hospital and late clinical outcomes and excellent 1-year patency for vein grafts anastomosed both by the St Jude Medical second-generation aortic connector system and by hand. The patency of the connector grafts did not differ from that of the hand-sutured grafts. (J Thorac Cardiovasc Surg 2010;139:741-7)
\end{abstract}

Vascular anastomoses in coronary artery bypass grafting (CABG) are usually performed with continuous sutures for both proximal and distal anastomoses. Several years ago, however, considerable interest arose in the development of sutureless anastomotic devices for CABG, spurred in part by increasing use of off-pump coronary artery bypass (OP$\mathrm{CAB}$ ) and the desire to avoid aortic manipulation and the side-biting clamp, as well as in their potential to facilitate minimally invasive surgery. The introduction of the Symmetry first-generation aortic connector (St Jude Medical, Inc, St

\footnotetext{
From the Department of Cardiothoracic Surgery, ${ }^{a}$ Sahlgrenska University Hospital, Göteborg, Sweden; the Department of Cardiothoracic Surgery, ${ }^{\mathrm{b}}$ Ceske Budejovice Hospital, Ceske Budejovice, Czech Republic; and the Division of Cardiovascular Surgery, ${ }^{\mathrm{c}}$ Peter Munk Cardiac Center, Toronto General Hospital, Toronto, Ontario, Canada.

Disclosures: This study was supported by an unrestricted grant from St Jude Medical, Inc, St Paul, Minn. Terrence M. Yau is supported by the Angelo and Lorenza DeGasperis Chair in Cardiovascular Surgery Research.

Read at the Eighty-eighth Annual Meeting of The American Association for Thoracic Surgery, San Diego, Calif, May 10-14, 2008.

Received for publication June 2, 2008; revisions received Sept 8, 2009; accepted for publication Sept 28, 2009.

Address for reprints: Terrence M. Yau, MD, MSc, Division of Cardiovascular Surgery, Peter Munk Cardiac Centre, Toronto General Hospital, 4 N-470, 200 Elizabeth St, Toronto, Ontario, Canada (E-mail: terry.yau@uhn.on.ca).

$0022-5223 / \$ 36.00$

Copyright (c) 2010 by The American Association for Thoracic Surgery

doi:10.1016/j.jtcvs.2009.09.063
}

Paul, Minn) was associated with satisfactory short-term outcomes, ${ }^{1-4}$ resulting in more than 40,000 implants worldwide. Further investigation revealed no effect of the device and delivery system on graft endothelial function ${ }^{5}$ and a 1-year patency rate of $100 \%$ in grafts to the right coronary artery. ${ }^{5}$ There were, however, reports of complications such as stenoses in the vein graft close to the connector site.$^{6-8}$ There were several issues with the first-generation Symmetry device, one being the requirement for a $90^{\circ}$ takeoff of the saphenous vein graft from the aorta, resulting in a potential risk of kinking if the proximal anastomosis was incorrectly sited. Another issue was the need for introduction of a transfer sheath into the vein graft, with at least the potential for endothelial injury. ${ }^{9}$

So that these issues could be addressed, a second-generation device was developed that allowed angled anastomoses and did not require instrumentation of the retained segment of the vein graft. The aim of this multicenter prospective randomized trial was to investigate the safety and efficacy of this second-generation anastomotic connector system.

\section{METHODS}

This study was approved by the local Ethics Committees of Sahlgrenska University Hospital, Toronto General Hospital, and Ceske Budejovice Hospital. 


\section{Abbreviations and Acronyms \\ $\mathrm{CABG}=$ coronary artery bypass grafting \\ $\mathrm{CT}=$ computed tomography \\ LITA $=$ left internal thoracic artery \\ MRI = magnetic resonance imaging \\ $\mathrm{OPCAB}=$ off-pump coronary artery bypass}

\section{Study Design}

This randomized trial was performed at 3 centers from August 2003 to December 2004. Patients undergoing elective or nonemergency isolated on-pump CABG with at least 2 planned saphenous vein grafts were included in the study. Exclusion criteria included the following: requirement for concomitant valve surgery, reoperative or emergency coronary surgery, OP$\mathrm{CAB}$, minimally invasive vein harvesting, left ventricular ejection fraction less than $25 \%$, creatinine level greater than $2.0 \mathrm{mg} / \mathrm{dL}$, current smoker, pregnancy, and participation in other investigational protocols. $\mathrm{Pa}-$ tients with saphenous veins that were too small or too large for the range of devices were excluded intraoperatively from the study. Patient demographics, intraoperative data, hospital outcomes, and clinical follow-up data were collected. Graft patency was assessed at 1 year by conventional contrast angiography, computed tomographic (CT) angiography, or magnetic resonance imaging (MRI).

\section{Operative Technique and Randomization}

The operation was performed by conventional on-pump techniques, with the use of a single crossclamp or side-biting clamp at the discretion of the surgeon. Epiaortic echocardiography was used to confirm that there was no significant aortic atherosclerosis at the proposed site of the proximal graft anastomoses. After construction of one or more distal anastomoses, the vein graft segments were cut to an appropriate length and the internal diameter of the proximal end of the vein graft was measured with a sizing tool. The appropriate size of the connector $(3.0-4.5 \mathrm{~mm}$ internal diameter) for that vein segment was recorded. Each graft/target vessel combination was assigned a number by the surgeon. After this, the randomization envelope for each graft number was opened. In this way, each proximal anastomosis was randomized, within each patient, to be constructed by the connector or by suture. Each patient received at least 1 connector graft and 1 hand-sutured graft. In patients receiving 3 or more vein grafts, it was left to the surgeon's discretion whether the third or fourth vein grafts were randomized or were performed in the standard fashion (and thus excluded from the comparative analysis).

Intraoperative graft flow evaluations were performed with an $8-\mathrm{MHz}$ Doppler flow meter with a corresponding size of flow probe (Medi-Stim Butterfly Flow Meter; Medi-Stim AS, Oslo, Norway) or by continuouswave Doppler sonography, according to usual practice at each institution.

All patients received acetylsalicylic acid postoperatively.

\section{Design and Delivery of the St Jude Medical Second- Generation Connector}

The design and delivery technique have been described elsewhere. ${ }^{8,10}$ In brief, the device is constructed of nitinol but was designed, in contrast to the first-generation device, to create an anatomically side-to-side, but functionally beveled, anastomosis. The graft is loaded onto the delivery system through the proximal end of the vein, which is then excluded from the graft after completion of the anastomosis, so that there is no contact of the delivery system with the endothelium of the retained segment of vein (Figure 1). After creation of the aortotomy and insertion of the connector, the connector is deployed and the delivery system removed. The proximal vein graft stump is ligated, with care taken not to create a residual stump (Figure 2).

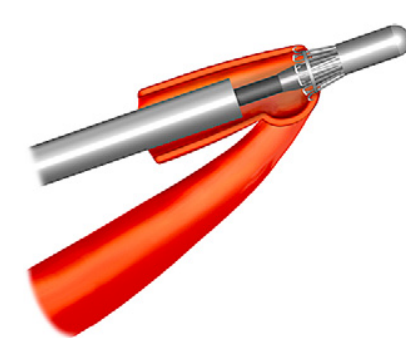

A

FIGURE 1. Schema showing loading (A) and placement (B) of the St Jude Medical second-generation aortic connector. A, The delivery system is introduced through the proximal end of the graft, which will subsequently be excluded. B, The device has hooks to engage the edges of the venotomy. The system is oriented perpendicularly to the aortotomy. (Reprinted with permission of the Cardiovascular Division, St Jude Medical, Inc, St Paul, Minn.)

\section{Follow-up and Graft Assessment}

Patients were followed up clinically at 6- and 12-month intervals. One year postoperatively, patients underwent radiographic evaluation of graft patency. For the majority of patients, this was carried out by contrast angiography of the vein grafts; angiography of left internal thoracic artery (LITA) and radial artery grafts was not mandated by the protocol. However, in the latter portion of the study, graft evaluation was performed by CT angiography or MRI to eliminate the small but nontrivial risks associated with contrast angiography. Graft patency and stenoses were evaluated by an independent core laboratory (PERFUSE Angiographic Core Laboratory and Data Coordinating Center, Boston, Mass).

\section{Statistical Analysis}

Data are presented as mean $\pm \mathrm{SD}$. Discrete data were subjected to $\chi^{2}$ analyses, whereas continuous data were analyzed by the Student $t$ test. Graft-related outcomes were analyzed on an intent-to-treat basis.

\section{RESULTS}

\section{Patients and Grafts}

A total of 151 patients with a mean age of $65 \pm 9$ years who met study inclusion/exclusion criteria were enrolled

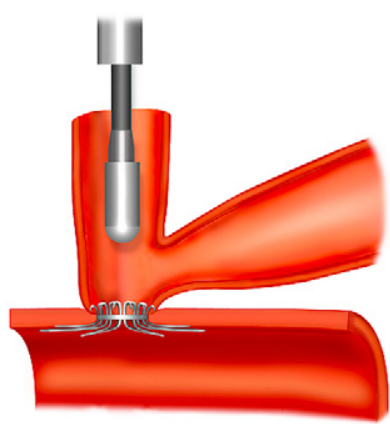

A

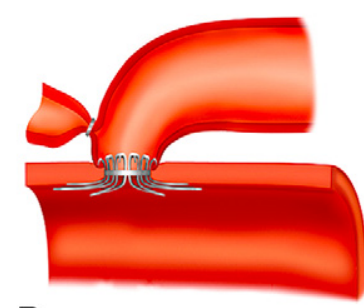

B
FIGURE 2. Schema showing disengagement of the delivery system from the deployed device (A) and final appearance of the proximal anastomosis (B). The side-to-side anastomosis is followed by careful placement of a clip to exclude the proximal segment of vein, rendering this into a functionally beveled anastomosis, and in which the endothelium of the retained vein graft segment has not been in contact with the delivery system. (Reprinted with permission of the Cardiovascular Division, St Jude Medical, Inc, St Paul, Minn.) 
TABLE 1. Patient demographics

\begin{tabular}{|c|c|c|c|c|}
\hline Variable $(\%)$ & $\begin{array}{l}\text { All patients } \\
(\mathbf{n}=\mathbf{1 5 1})\end{array}$ & $\begin{array}{c}\text { Patients undergoing } \\
\text { graft evaluation }(n=120)\end{array}$ & $\begin{array}{l}\text { Patients not undergoing } \\
\text { graft evaluation }(n=31)\end{array}$ & $\begin{array}{c}P \text { value for } 120 \text { patients } \\
\text { vs } 31 \text { patients }\end{array}$ \\
\hline Male & 87 & 87 & 87 & .9 \\
\hline Hypertension & 63 & 63 & 65 & .8 \\
\hline Hypercholesterolemia & 64 & 64 & 61 & .8 \\
\hline Previous TIA & 3.3 & 4.2 & 0 & .2 \\
\hline Previous CVA & 2 & 1.7 & 3.2 & .6 \\
\hline PVD & 7.3 & 5.8 & 13 & .2 \\
\hline Smoking history & 60 & 58 & 71 & .2 \\
\hline Diabetes & 33 & 33 & 32 & 1.0 \\
\hline Renal failure & 2.7 & 1.7 & 6.5 & .1 \\
\hline Previous MI & 54 & 57 & 45 & .3 \\
\hline Congestive heart failure & 4.6 & 5 & 3.2 & .7 \\
\hline Angina & 92 & 93 & 90 & .7 \\
\hline Unstable angina & 53 & 56 & 42 & .2 \\
\hline CCS angina class & & & & .1 \\
\hline I & 19 & 19 & 16 & \\
\hline II & 18 & 18 & 16 & \\
\hline III & 37 & 37 & 39 & \\
\hline IV & 24 & 24 & 23 & \\
\hline NYHA functional class & & & & .9 \\
\hline I & 47 & 48 & 31 & \\
\hline II & 24 & 24 & 45 & \\
\hline III & 13 & 13 & 23 & \\
\hline IV & 2 & 1.7 & 3.2 & \\
\hline Previous PTCA & 14 & 13 & 16 & .7 \\
\hline Previous stent & 6 & 5.8 & 6.5 & .9 \\
\hline
\end{tabular}

TIA, Transient ischemic attack; $C V A$, cerebrovascular accident; $P V D$, peripheral vascular disease; $M I$, myocardial infarction; $C C S$, Canadian Cardiovascular Society; $N Y H A$, New York Heart Association; PTCA, percutaneous transluminal coronary angioplasty.

in the study and were analyzed. Their demographics are detailed in Table 1. A total of 489 grafts were constructed in these patients $(3.2 \pm 0.5$ grafts per patient), including 327 vein grafts randomized to the connector $(n=162)$ or suture $(n=165)$. There were also 140 LITA grafts, 2 radial artery grafts, and 20 saphenous vein grafts that were not randomized (some patients had more than 2 vein grafts, but only 2 vein grafts were randomized).

Connector implantation. In 162 connector grafts, 151 (93.2\%) devices were successfully implanted on the first attempt. Technical problems, including excessive gathering of the side walls of the vein graft causing a "seagull", deformity of the hood of the graft, required explantation of 11 devices intraoperatively. A second device was used in 6 of these grafts, for a total successful connector implantation rate of $96.9 \%$, and the other 5 grafts were sutured.

In-hospital outcomes. There were $2(1.3 \%)$ early deaths. One death occurred in a 79-year-old man with insulin-dependent diabetes and a left ventricular ejection fraction of $40 \%$, in whom protamine administration after cardiopulmonary bypass was associated with cardiovascular collapse and in whom attempts at resuscitation were unsuccessful. The other death occurred in an 83-year-old patient in whom the initial connector deployment resulted in a visually obvious moderate stenosis at the proximal anastomosis. The connector was replaced with a second device without incident. The hemodynamic course in this patient was unremarkable, but both respiratory and dialysis-dependent renal failure developed. The family opted for palliative care rather than continuing dialysis, and the patient died several weeks after hospital discharge.

Five $(3.3 \%)$ patients underwent reoperation for bleeding, including 1 patient who had re-exploration twice. In none of these patients was a connector graft the source of bleeding. No patient underwent reoperation for graft revision. Four $(2.6 \%)$ patients had strokes and $1(0.7 \%)$ patient had a transient ischemic attack. One $(0.7 \%)$ patient had a perioperative myocardial infarction. Two $(1.3 \%)$ patients had sternal osteomyelitis. Two $(1.3 \%)$, patients had renal failure requiring dialysis postoperatively, and another $2(1.3 \%)$ had elevated creatinine levels but did not require dialysis. Thirty-seven $(25 \%)$ patients had atrial fibrillation postoperatively.

Midterm outcomes. One $(0.7 \%)$ patient, a 51-year-old man with a left ventricular ejection fraction of $35 \%$ who had had an uneventful hospital course, died 10 months postoperatively. A postmortem examination demonstrated a posterior myocardial infarction, with all 3 of the bypass grafts patent. 
One $(0.7 \%)$ patient had an occipital stroke during followup angiography. Because of this event, subsequent patients were evaluated by CT angiography or MRI.

At 1 year, patients reported a mean Canadian Cardiovascular Society angina class of $1.01 \pm 0.2$.

Graft evaluation and patency. Patency was evaluated in $120(81 \%)$ patients with 260 study grafts. Of the 120 patients returning for graft assessment, 74 patients with 161 grafts were evaluated by coronary angiography, 31 patients with 69 grafts by MRI, and 15 patients with 30 grafts by CT. Demographics and intraoperative variables for the $120 \mathrm{pa}-$ tients undergoing graft assessment versus the 31 patients who did not are compared in Table 1. The mean interval from surgery to angiographic/MRI/CT follow-up was 418 \pm 83 days (13.9 months).

The 1-year patency rate for study grafts constructed with the anastomotic connector was $92.2 \%(118 / 128)$ and for hand-sutured grafts, $91.7 \%$ (121/132).

Analysis of various subgroups in the 120 patients undergoing graft evaluation demonstrated patency rates of $92.9 \%$ (13/14 grafts) in nonrandomized saphenous vein grafts and of $90 \%$ (9/10 grafts) in grafts randomized to the connector, in which the first connector had to be explanted immediately owing to technical issues. There were 113 LITA grafts in the 120 patients undergoing graft evaluation. The LITA was evaluated in 78 of those patients, with an observed patency rate of $96.2 \%(75 / 78)$.

Five $(1.8 \%)$ nonocclusive stenoses were observed in the 274 vein grafts evaluated. There was 1 stenosis, of $50 \%$ severity, in the distal portion of a sutured vein graft. There were $4(3.1 \%)$ stenoses, all ostial in location, in the 128 connector grafts, ranging from $65 \%$ to $90 \%$ in severity. All 4 of these patients were free of angina or reintervention at 1-year follow-up.

\section{DISCUSSION}

This prospective randomized multicenter study demonstrated excellent patency, at more than 1 year, of grafts in which the proximal anastomoses were constructed with a second-generation anastomotic connector. Patency rates of conventional sutured grafts were similarly excellent. Use of this second-generation device therefore had no discernible impact on midterm graft patency.

Rates of mortality and major morbidity were in line with current expectations for CABG surgery. As our within-patient randomization scheme meant that all patients had both connector and sutured grafts, it was not possible to compare mortality and morbidity between connector and suture groups. However, the 2 early deaths and 1 late death were unrelated to the device, or apparently to the grafts at all, and in the patients who underwent re-exploration for bleeding, there was no bleeding from any connector anastomoses. There were thus no significant safety issues identified with use of the device. There were, however, 11 connectors that were explanted after initial deployment owing to technical issues. Most of these issues occurred early in our experience.

The excellent patency rates of the second-generation connector observed in this study contrast with some reports of the experience with the first-generation Symmetry device. Whereas patency rates of as high as $100 \%$ at 1 year have been reported with first-generation connectors, ${ }^{5}$ stenoses in the proximal segment of the vein graft, in proximity to the connector, have been noted in $22 \%$ to $38 \%{ }^{5,6}$ of grafts. Others have noted significant rates of occlusion and reintervention associated with the first-generation device. ${ }^{7,8}$

At least 3 theories have been advanced as potential reasons for the higher-than-anticipated rates of proximal stenoses and occlusions in grafts in which the first-generation device was used. Differences between the first- and second-generation devices in these 3 aspects may therefore underlie any observed differences in patency. First, the original device created a $90^{\circ}$ end-to-side anastomosis. Particularly for left-sided grafts, it is easy to have a graft kinked proximally against the pericardium or mediastinal tissue, inasmuch as it does not adopt the usual course of a sutured graft with a beveled anastomosis. This problem is accentuated when proximal anastomoses are constructed with a partial-occlusion clamp, inasmuch as they are moved more anteriorly on the aorta. The second-generation device creates an anatomically side-to-side but functionally beveled anastomosis, avoiding this difficulty.

A second issue with the first-generation device was that its delivery system required instrumentation, with a transfer sheath, of the vein segment that was retained as a graft. Although ex vivo experiments did not demonstrate an obvious effect on endothelial function, ${ }^{5}$ it seems likely, particularly with small vein segments that were pulled onto the transfer sheath, that endothelial injury or denudation may have occurred. The delivery system for the second-generation device is passed through the vein through its proximal end, via a segment that is subsequently excluded with a clip. Therefore, the vein segment functioning as a graft is never instrumented and should not be subject to any additional endothelial insult.

A third but perhaps less important issue was that proximal anastomoses had to be constructed before distal anastomoses when the first-generation connector was used. This is not the usual order of anastomoses for most surgeons, and determination of the correct length of the vein, and avoidance of tension on the connector anastomosis, may be more difficult. The second-generation device permits construction of distal anastomoses before proximal anastomoses and thus requires less deviation from the usual routine.

From a practical point of view, the loading process for the second-generation device is also considerably simpler and faster, requiring only 10 to 15 seconds, than that for the original device. It is not as difficult to visually confirm that the vein is properly loaded. 
In this study, the 1-year patency of both the sutured and connector vein grafts was approximately $92 \%$. This compares favorably with other randomized trials in which vein graft patency was evaluated, but as in most studies involving novel intraoperative interventions and invasive follow up, these were highly selected patients. Our patency rates may have been influenced by our vein sizing criteria; only vein segments with internal diameters between 3.0 and $4.5 \mathrm{~mm}$ were used, excluding poor quality veins with varicosities or severe phlebitis. In practice, approximately $10 \%$ of vein segments were believed to be unsuitable; this was not a common phenomenon. Patients who agree to participate in a clinical trial may be more health-conscious and more likely to comply with antiplatelet and cholesterol-lowering medications, thereby influencing graft patency. Patients who agree to return for study angiography may be further self-selecting. However, the characteristics of the 120 patients returning for graft evaluation did not differ in any measured variable from the 31 who did not.

The patency rate was $93 \%$ in the small number of vein grafts that were not randomized, suggesting that the good patency of randomized grafts was not related to selection of optimal targets. The patency rate of LITA grafts was $96 \%$, consistent with expectations for a 1-year assessment. Importantly, in 10 grafts in which a connector had initially been implanted, but which had to be replaced with a second connector or converted to a sutured anastomosis owing to technical issues, patency was $90 \%$. Thus a failed or suboptimal initial deployment of the device was not associated with reduced patency at 1 year. We did note, however, a 3\% incidence of stenosis at or near the device in the connector grafts.

One patient had an occipital stroke during follow-up coronary angiography, as the LITA was being instrumented. This event led to a decision by the data safety and monitoring board to abandon any further conventional angiography and switch to CT angiography and MRI to assess graft patency. Although the risk of stroke during angiography is very low, its potential seriousness raises issues as to whether conventional angiography continue to be the standard technique for future graft patency studies. Given the continuing advances in CT angiography, this modality may become the de facto standard for investigation.

The history of the St Jude Medical aortic connector systems illustrates the complexity of the clinical introduction of new technology and the importance of controlled studies when evaluating new technical equipment in medicine. After promising feasibility studies with short periods of follow-up without controls, the first-generation connector was widely used and more than 40,000 implants were performed worldwide. After some time, it became clear that there were questionable issues with regard to graft patency with the connector-made anastomosis, and users began to lose confidence in the technology. Reported results with the first-generation device varied from excellent to poor. In addition to the factors detailed above, it became clear that extreme care was required during loading of the vein segments onto the delivery system, and variability in this aspect may also have contributed to the discrepant results. Ultimately, owing to the emergence of drug-eluting stents and other factors, there was a decline in the volumes of CABG and a decreasing interest in OPCAB, which may have been the main market for the device. The decrease in the market for the device led to St Jude Medical making the decision in September 2004 to discontinue manufacture and sales of the device. Before that time, developmental work had resulted in the second-generation device and the multicenter study that had already been initiated.

In conclusion, this prospective multicenter randomized controlled trial demonstrated good in-hospital and late clinical outcomes and excellent 1-year patency for vein grafts anastomosed both by the St Jude Medical second-generation aortic connector system and by conventional suture techniques. The patency of the connector grafts did not differ from that of the hand-sutured grafts. Nonrandomized vein grafts and LITA grafts also had excellent patency rates.

\section{References}

1. Eckstein FS, Bonilla LF, Englberger 1, Stauffer E, Berg TA, Schmidli J, et al. Minimizing aortic manipulation during OPCAB using the symmetric aortic connector system for proximal vein graft anastomoses. Ann Thorac Surg. 2001;72(Suppl): S995-8.

2. Eckstein FS, Bonilla LF, Englberger L, Immer FF, Berg TA, Schmidli J, et al. The St Jude Medical symmetry aortic connector system for proximal vein graft anastomoses in coronary artery bypass grafting. J Thorac Cardiovasc Surg. 2002;123: 777-82.

3. Wiklund L, Bugge M, Berglin E. Angiographic results after the use of a suturless aortic connector for proximal vein graft anastomoses. Ann Thorac Surg. 2002;73: 1993-4

4. Mack MJ, Emery RW, Ley LR, Cole PA, Leonard A, Edgerton JR, et al. Initial experience with proximal anastomoses performed with a mechanical connector. Ann Thorac Surg. 2003;75:1866-71.

5. Verma S, Fedak PW, Ko L, Cusimano RJ, Walton NA, Parker JD, et al. Evaluation of a novel sutureless anastomotic connector: from endothelial function to mid-term clinical and angiographic follow-up. J Thorac Cardiovasc Surg. 2003;126:1555-60.

6. Carrel TP, Eckstein FS, Englberger L, Windecker S, Meier B. Pitfalls and key lessons with the symmetry proximal anastomotic device in coronary artery bypass surgery. Ann Thorac Surg. 2003;75:1434-6.

7. Traverse JH, Mooney MR, Pedersen W. Clinical, angiographic, and interventional follow-up of patients with aortic-saphenous vein graft connectors. Circula tion. 2003;108:452-6.

8. Carrel TP, Eckstein FS, Englberger L, Berdat PA, Schmidli J. Clinical experience with devices for facilitated anastomoses in coronary artery bypass surgery. Ann Thorac Surg. 2004;77:1100-20.

9. Farhat F, Chalabreysse L, Diab C, Aubert S, Jegaden O. Histological aspects of the saphenous vein damage with the use of the symmetry aortic connector system. Interact Cardivasc Thorac Surg. 2004;3:373-5.

10. Setina M, Krchnakova A, Mokracek A, Pesl L, Bonilla LF. The second-generation aortic connector: six months angiographic follow-up. Ann Thorac Surg. 2005;80: 695-9.

\section{Discussion}

Dr John W. Hammon, Jr (Winston-Salem, NC). This study was a continued analysis of data that were presented at this meeting last year. That study also showed that brain injury was magnified by 
rewarming whereas the brain was not overtly protected by maintaining the patient at a hypothermic temperature, thus incriminating rewarming in the injury profile of two different organ systems. For the benefit of any perfusionists present and those of us who have been studying this, I would like to ask you a couple of questions.

You mentioned that rate of rewarming has been shown to be important. Was rate of rewarming standard in your study or was it determined by the perfusionist or the surgeon, and did you find any correlation between rate of rewarming and renal injury?

Dr Boodhwani. Thank you very much for those positive comments, Dr Hammon. The rate of rewarming was controlled only by the fact that patients enrolled in this trial were relatively uniform. They all underwent straightforward, nonemergency CABG surgery. In those patients we examined the time period over which the warming occurred, and typically it occurred over a period of 20 to 25 minutes. The temperature change in the rewarmed group was from $32{ }^{\circ} \mathrm{C}$ to $37^{\circ} \mathrm{C}$. So that was approximately the rate of rewarming. This was tracked in individual patients but specific correlations for renal function were not performed.

Dr Hammon. The second question relates to the actual temperature of the arterial blood entering the patient's ascending aorta through the perfusion cannula. In your manuscript you state that the temperature was never allowed to rise above $37.5{ }^{\circ} \mathrm{C}$. First, where exactly was that measured? Second, what was the water bath temperature that the perfusionist set in the pump to achieve that temperature? In many cases the water bath temperature is as high as $39^{\circ} \mathrm{C}$, which can have differential heating effects on the blood.

Dr Boodhwani. The study protocol did not control exactly the water bath temperature. It was controlling the oxygenator outlet temperatures, though, and oxygenator outlet temperatures were reflective of the temperature at which the blood enters the patient's body. Those were never allowed to exceed $37.5^{\circ} \mathrm{C}$ in the normothermic patients and $34.5^{\circ} \mathrm{C}$ in the patients assigned to mild hypothermia.

Dr Hammon. I have one more question, and it is really more of a hypothesis than anything else. This morning we heard in an offpump study that there is much more elaboration of myocardial enzymes in patients undergoing on-pump surgery than off-pump surgery. Could it be elaborated as a hypothesis that one of the reasons for this would be that the 3 organs in the body that get the most blood flow during rewarming - the brain, the kidneys, and the heart-have now been shown to manifest injury? At least 2 of them have. Could this increased elaboration of enzymes from the heart also be due to rewarming, and did you collect enzyme data on your patients that you could study and confirm my humble hypothesis?

Dr Boodhwani. That is actually a very insightful hypothesis. We did collect cardiac enzyme data in these studies, and we found that the normothermic patients had higher levels of creatinine kinase. Troponin T levels were only measured in our cohort if the creatinine kinase levels exceeded $800 \mathrm{mg} / \mathrm{dL}$, and troponin levels in fact were not significantly different in the study. Having said that, the only additional thing that I would point out is that there is a key difference between the heart and the other organs: obviously, the heart undergoes a definite period of ischemia and reperfusion, whereas the mechanisms of renal or brain injury that occurs with cardiopulmonary bypass still remain to be clearly defined, whether it is an ischemic burden or an inflammatory injury. Many hypotheses have been postulated, and we are still unclear what the exact effect of temperature is on those mechanisms of injury.

Dr Christina Mora-Mangano (Stanford, Calif). This is an elegant paper, and your group has done a number of important studies on temperature management and end organ dysfunction in patients undergoing cardiac surgery. However, there are potential problems regarding your definition of renal dysfunction. The nephrology community has been challenged to agree on a definition of renal dysfunction. The definition applied in this study differs from that used by The Society of Thoracic Surgeons database and is not consistent with definitions that we have used in some of our work. In your report, $17 \%$ of the study patients had postoperative renal dysfunction; this is substantially greater than rates reported in the majority of studies assessing perioperative renal function. Most investigations suggest that $5 \%$ to $8 \%$ of cardiac surgery patients will have an important compromise in renal function. Can you address the issues regarding the definition of renal dysfunction? Additionally, in view of the "inclusive" definition of dysfunction, was there a significant increase in the duration of hospitalization for those patients characterized as having an adverse renal event?

Dr Boodhwani. Thank you for those very good questions. You are right. The definition of postoperative renal dysfunction is significantly debated in the literature. Older studies have used one of two clinical measures, the first one being postoperative serum creatinine levels and the second being estimated creatinine clearance using a variety of different formulas. The incremental increase that constitutes significant renal dysfunction is variable in the literature. However, a common cutoff that has been used is $25 \%$. A number of studies have correlated this degree of renal dysfunction with hard clinical end points of mortality, morbidity, as well as intensive care unit and hospital stay.

As you mentioned, there was a recent consensus conference by the Acute Dialysis Quality Initiative to more clearly define renal dysfunction, and they came up essentially with 3 recommendations. The first one was that calculated creatinine clearance or urinary protein measurement should not be used. The second recommendation was that estimated creatinine clearance should be incorporated into the definition, if possible. The third recommendation was that change from baseline is a better indicator of renal dysfunction than absolute values at any given point in the postoperative period. We tried to incorporate all these aspects of the definition, and in fact the risk factor and life expectancy criteria that were described in that Acute Dialysis Quality Initiative report suggested that a $25 \%$ reduction in estimated creatinine clearance would constitute mild renal injury.

Having said that, we looked at our cohort and recognized that this is a relatively low-risk population. Had we chosen a very stringent definition of renal dysfunction, you are right, our renal dysfunction rates would have been much lower, but we probably would have missed a clinically significant effect of the temperature intervention. That is why we chose an end point that was fairly frequent in our study population but met the criteria for clinical significance.

To answer the second part of your question, patients who had renal dysfunction in our study did stay in the hospital an average of 2 
days longer than the patients who did not. This has some impact on clinical outcome.

Dr Mora-Mangano. I would like to make one more comment about the question regarding the rate of rewarming after hypothermic cardiopulmonary bypass. I believe that we still lack the data to assess the impact of the rate of warming on perioperative adverse outcomes. Currently available studies should undergo careful scrutiny. In most reports, subjects in the rapid-rewarming group achieve greater absolute temperatures than do those patients rewarmed more slowly. Thus it is unclear as to whether or not we are assessing and comparing the actual rewarming rate or the highest temperature achieved in the study groups.

Dr Bruce B. Davis (Malvern, Australia). I have enjoyed your work over the years. Your maximum blood temperature of $37.5^{\circ} \mathrm{C}$ seems to be higher than what we would have used, and I am not sure I picked up your incremental rate of warming. Can you comment on that first?

Dr Boodhwani. As a pretext to these studies, we conducted a survey of various Canadian centers looking at temperature practices and found that in some cases people exceed $37.5^{\circ} \mathrm{C}$. This sur- vey was conducted in the 1990 s, and perhaps that information is a bit outdated. But about $30 \%$ of centers at that time were exceeding temperatures of $38^{\circ} \mathrm{C}$. We believed at the time of the design of these studies that $37.5^{\circ} \mathrm{C}$ was a safe temperature to be the ceiling temperature that we would permit in these trials.

Dr Davis. And the incremental rate of warming?

Dr Boodhwani. By that you mean the rate over which the temperature changes?

Dr Davis. Yes.

Dr Boodhwani. In this study that occurred over a period of 20 to 25 minutes for a $5^{\circ} \mathrm{C}$ change in temperature.

Dr Davis. Did you have any problems with postoperative and intensive care, which we would have if we did not rewarm the patients? Were they intubated for much longer?

Dr Boodhwani. That is a great point. In the second study in which sustained hypothermia was compared with normothermia, there was in fact a trend toward sooner discharge from the intensive care unit in the hypothermic patients. So actually there was no detrimental effect of hypothermia in terms of length of ventilation or length of intensive care unit stay, bleeding, or infection rates. 